\title{
El proceso de evaluación del aprendizaje desde el Entorno Virtual de Aprendizaje en el nivel universitario
}

Fecha de recepción : 2021-06-10 • Fecha de aceptación: 2021-08-13 • Fecha de publicación: 2021-09-10

\author{
Arian Fuentes Aparicio' \\ Colegio Bilingüe Almirante Nelson, Ecuador \\ arian.fuentes@ueanan.edu.ec \\ https://orcid.org/0000-0002-2633-2244 \\ Betty Pastora Alejo² \\ Universidad Tecnológica Israel, Ecuador \\ bettyalejo2012@gmail.com \\ https://orcid.org/0000-0002-9837-3264
}

Ariadna Granados Campo²

Universidad Tecnológica Israel, Ecuador

agranados@uisrael.edu.ec

https://orcid.org/0000-0002-5991-7783

Osley Puerto Menéndez ${ }^{3}$

Unidad Educativa Particular Glenn Doman, Ecuador

osleypuerto@glenndomanmanta.edu.ec

https://orcid.org/0000-0002-6981-4374

\section{Resumen}

La evaluación del aprendizaje desde el entorno virtual alcanza gran connotación en el contexto universitario actual, teniendo en cuenta que esta posibilita la comunicación y constatación de resultados, ya sea de manera sincrónica o asincrónica. Requiere la atención de todos en un nuevo escenario pedagógico al reconocer que existe una gran diferencia entre los procesos evaluativos, en este contexto, y en los ambientes de aula con carácter presencial. Tomando estas premisas como 
punto de partida, en el presente artículo se define como objetivo general el reconocer la importancia de la evaluación del aprendizaje desde el Entorno Virtual de Aprendizaje en el contexto universitario actual. Se emplearon diferentes métodos, entre los que se encuentran el nivel teórico, método analítico - sintético; del nivel empírico, revisión documental y la encuesta; fue empleado además el análisis porcentual del nivel estadístico y del nivel lógico de la investigación, el método inductivo. En un primer momento el proceso investigativo tuvo lugar por medio de la investigación documental. Posteriormente fue practicada una encuesta a docentes seleccionados para corroborar los resultados alcanzados, dando cumplimiento al objetivo general propuesto.

\title{
Palabras clave: evaluación del aprendizaje, entorno virtual de aprendizaje.
}

\begin{abstract}
The evaluation of learning from the virtual environment reaches great connotation in the current university context, considering that it enables communication and verification of results, either synchronously or asynchronously. It requires everyone's attention in a new pedagogical scenario, recognizing that there is a great difference between the evaluation processes in this context and in classroom environments with a face-to-face character. Taking these premises as a reference, this article defines the general objective of recognizing the importance of learning assessment from the Virtual Learning Environment in the current university context. During the research process, different methods were used, among which are the theoretical level, the analytical-synthetic method; the empirical level, documentary review and survey; the statistical level, percentage analysis; and the logical level of the research, the inductive method. The research process was initially developed through documentary research. Subsequently, a survey was made to selected teachers to corroborate the results obtained, thus fulfilling the proposed general objective.
\end{abstract}

Keywords: learning assessment, virtual learning enviroment. 


\section{Introducción}

La evaluación es uno de los procesos de mayor importancia en los contextos educativos. A través de ella es posible medir los aprendizajes que logra el alumnado y así perfeccionar el proceso de enseñanza-aprendizaje. En la educación superior, tradicionalmente la evaluación ha estado centralizada de manera prioritaria sobre su función sumativa; es decir, orientada hacia la constatación de resultados y la medición de aprendizajes al término de un proceso de enseñanza. Cabe resaltar que en los últimos años se ha evidenciado el surgimiento de nuevos enfoques de evaluación, que intentan buscar alternativas en relación a las concepciones establecidas y prácticas evaluadoras tradicionales.

La evaluación del aprendizaje en el nivel universitario representa en la actualidad, una ocupación y preocupación que se refleja tanto en labor de los docentes en diferentes escenarios educativos. Basta solamente poner estos tres términos: evaluación - universidad - aprendizaje en cualquier buscador para quedar completamente sorprendido ante la cantidad de información que dicha búsqueda genera. Por otra parte, cuando se concreta ese ambiente evaluativo en el entorno virtual, la inquietud se hace aún mayor.

Ante la crisis sanitaria que ha vivido el mundo y que todavía persiste, los procesos de aprendizajes han variado significativamente, $y$, por ende, el proceso de evaluación académica se ha visto sujeto a nuevos retos, sobre los cuales Ecuador no ha estado exento. El uso necesario e inminente de las Tecnologías de la Información y las Comunicaciones (TIC) en la educación superior insiste en revisar las teorías y prácticas de la evaluación del aprendizaje o desempeño de los estudiantes, para comprobar su pertinencia y confiabilidad, o la necesidad de generar nuevos enfoques, como la evaluación en un ambiente virtual, con características diferentes a la evaluación tradicional.

Se debe añadir que la evaluación académica en la educación superior, desde un Entorno de Virtual de Aprendizaje (EVA), genera un proceso sistémico que implica que el profesor revise el modelo pedagógico que brinda desde sus clases, que aplique estrategias y herramientas que permitan constatar la evolución y el progreso real alcanzado por los alumnos. Es importante reconocer que existe una relación directa entre la planificación de la evaluación y la metodología de enseñanza empleada por los docentes. Depende en gran medida cómo sea concebida, ya que puede ser reconocida como un juicio o como una ocasión para aprender.

El cambio en los modelos educativos y en la evaluación es también una exigencia de la situación actual, que ubica a la enseñanza universitaria frente al reto de formar ciudadanos y futuros profesionales que puedan insertarse en diferentes contextos sociales y laborales que pueden ser objetos de constantes transformaciones, demostrando autonomía, flexibilidad y capacidad de adaptación. Esto exige enfoques de enseñanza que se apartan de los modelos tradicionalmente practicados en la Educación Superior. Sin duda, estos nuevos enfoques vienen aparejados de cambios en el proceso de evaluación académica.

Desde las nuevas perspectivas sobre la función de la evaluación, y tomando como premisa el escenario de cambio que se evidencia, constituye una necesidad reconsiderar el sentido de la evaluación en la enseñanza universitaria. Más que la comprobación final, es necesaria 
la evaluación continua del progreso de los estudiantes, centrando la atención en las tareas desarrolladas y también en las que el estudiante pudiera desarrollar en otros contextos educativos, como parte del estudio y la sistematización de una materia. Es preciso potenciar una suficiente retroalimentación, donde se apoye la mejora del aprendizaje, haciendo un uso adecuado de este proceso desde el EVA.

Teniendo en cuenta las diferentes perspectivas expuestas anteriormente, se pretende proporcionar un marco de reflexión centrado en el quehacer cotidiano basado en el trabajo docente, para ello se propone como idea a defender: la implementación efectiva del proceso evaluativo en el nivel universitario desde cómo el EVA contribuye a un desarrollo intelectual de calidad en los estudiantes.

Se toma esta idea como eje principal en la investigación y se destaca la necesidad de reconocer que la docencia no puede quedarse fuera de los contextos sociales, no se puede olvidar la dificultad de incorporar las TIC al ejercicio docente, pero esto ha de hacerse desde la primacía de lo educativo, lo que trae consigo la necesidad de considerar un marco general para comprender mejor la práctica de la evaluación académica.

\subsection{La evaluación del aprendizaje}

Varios son los autores que han aportado su criterio en el plano de la teoría sobre la evaluación del aprendizaje. Algunos coinciden al manifestar que constituye el escenario donde el evaluador emite un juicio de valor sobre un aspecto de la realidad que se pretende cambiar. Esta es un proceso que puede estar constituida por tres momentos: recogida de información, análisis de dicha información y juicio sobre el resultado de éste; y toma de decisiones a partir del juicio aportado (Jorba \& Sanmartí, 1996).

Desde una visión actualizada, la evaluación surge como un instrumento que es implementado en el proceso de enseñanza - aprendizaje, integrada en la labor cotidiana del docente. Además, debe ser el punto de partida en la toma de decisiones que afectan a la intervención educativa, al mejoramiento del proceso y a la instauración de medidas de refuerzo académico o de adaptación del currículo.

Se puede inferir que permite el cambio educativo y el desarrollo profesional de los docentes. Si se parte del hecho de que el hombre es un ser ético, esta condición lleva aparejada, inseparablemente, la capacidad de emitir juicios con un carácter asiduo. Es por ello por lo que la evaluación es una actividad que se realiza a diario. Sobre esto, se debe comprender que la evaluación, como parte del quehacer humano, se desarrolla por personas y afecta o beneficia directamente a personas (Zaragoza, 2003).

Aunque se conoce que la evaluación es un proceso que se implementa en muchos, por no decir todos, escenarios de la vida cotidiana de los seres humanos, al abordar la definición de evaluación, se figura en la mente de todos el mundo escolar y educativo. (Castillo \& Cabrerizo, 2003). 
Otros expertos en el tema centran su atención en evaluar diferentes procedimientos para valorar sus ventajas y desventajas en la evaluación de los alumnos. Es por ello lo que algunos autores manifiestan seguir los criterios de validez, confiabilidad e impacto deseado, añadiendo que los procedimientos que se pueden desarrollar durante este proceso tienen más garantías de éxito al realizarse internamente en las propias instituciones, que cuando son impuestos por agentes externos (Ploegh et al., 2009).

Las definiciones anteriores de la evaluación se ajustan a una concepción que se podría determinar cómo constructivista, actual o auténtica del aprendizaje, la propia evaluación y de la educación en general. Esta concepción sostiene que la evaluación no es un acto aislado, sino un proceso que no afecta sólo a los alumnos, sino a todos los elementos que forman parte del escenario educativo.

Desde esta perspectiva no se pretende mejorar sólo el aprendizaje actual, sino también el futuro, a través de evaluaciones integradoras que incluyan la valoración de todo el proceso de enseñanzaaprendizaje, y la consideración de todos sus actores, tanto estudiantes, como profesores, en lo individual y lo grupal.

También se puede ver la evaluación como actividad de aprendizaje en la medida en que por ella se adquiere conocimiento, llegando a asegurar que sólo cuando garantice el conocimiento, se puede garantizar la evaluación, la buena evaluación transformada ella misma en medio de aprendizaje y expresión del conocimiento (Bélair, 2000).

A partir de las ideas expuestas, se evidencia la importancia de la evaluación, pues se conforma como un elemento principal en el contexto educativo, al constituir la herramienta de seguimiento del proceso de enseñanza-aprendizaje, lo que permite la obtención de información sobre cómo se desarrolla dicho proceso para ajustar la intervención educativa, en función de los datos obtenidos. En resumen, es el elemento que retroalimenta el proceso de enseñanza-aprendizaje.

En tal sentido, si la evaluación tiene como meta fundamental asegurar el progreso de los estudiantes en la construcción de su aprendizaje, a la pregunta de qué evaluar se habría de responder expresando que todos los aspectos presentes en el proceso de enseñanza-aprendizaje y aquellos que, sin formar parte directa de dicho proceso, interfieren en él.

\subsection{La evaluación del aprendizaje según su funcionalidad u objetivo}

Desde la revisión bibliográfica se pudo comprobar que existen diferentes criterios de clasificación de la evaluación. Sobre esto se comprobó que, para algunos autores, la evaluación del aprendizaje se presenta en función de dimensiones tales como: la funcionalidad u objetivo de esta y la temporalidad (Casanova, 2012).

En la presente investigación se asume y se analiza la evaluación del aprendizaje según su funcionalidad u objetivo, por ser de interés de los investigadores, entre las que se encuentran: la evaluación diagnóstica, sumativa y formativa. 
La evaluación diagnóstica se desarrolla al principio de cada periodo académico y se emplea para que los profesores reconozcan el nivel de competencias que muestran los alumnos al comenzar el proceso de enseñanza - aprendizaje, posibilitando que ejecute una planificación acertada y lo orienta teniendo en cuento las necesidades de cada uno de los estudiantes; se considera el punto de partida para organizar el trabajo a lo largo del periodo académico.

La figura principal durante la realización de la evaluación es el docente, ya que partiendo del conocimiento que posee de sus estudiantes, diseña, organiza, coordina, orienta y da seguimiento a las acciones y actividades a realizar en las clases para el logro de las competencias, sabe las necesidades y las dificultades a las que se enfrentan sus alumnos. Se concibe este proceso como aquel acto cuando se juzga con anterioridad lo que transcurrirá durante el acto educativo o después de este (Martínez, 2009).

Al considerar este tipo de evaluación se recomienda el empleo de instrumentos como medio de obtención de información: pruebas objetivas estructuradas, explorando o reconociendo la situación real de los alumnos en relación con el hecho educativo.

Algunas de sus características son:

- Permite conocer el nivel de conocimientos previos que sirven como puntos de partida para la adquisición de nuevos aprendizajes.

- Detecta el nivel real del estudiante, con el fin de establecer actividades y métodos de enseñanza.

- Mide conductas de entrada cognitiva que posee cada alumno.

- Los resultados deben ser comunicados mediante la retroalimentación, enfatizando en los logros y deficiencias.

Siguiendo el curso del análisis sobre la temática en cuestión, se enfatiza en la evaluación formativa, reconociendo que su propósito es la toma de decisiones sobre las alternativas de acción y dirección que aparecen durante el avance del proceso de enseñanza-aprendizaje. La función fundamental es la conducción del aprendizaje para obtener mejores resultados. Se implementa durante todo el proceso educativo, o en cualquiera de los puntos conflicto del proceso. Dentro de éste, se pueden emplear diferentes instrumentos que sirven de apoyo a la evaluación: pruebas informales, observación y registro del desempeño, interrogatorios, entre otros.

Se debe resaltar que se emplea para dar seguimiento al avance del aprendizaje y facilitar retroalimentación al estudiante sobre sus logros, dificultades y posibilidades de avance. Es un momento mediante el cual se recauda información sobre el proceso de enseñanza aprendizaje, que facilita a los docentes la toma de decisiones sobre cómo enseñar, y los alumnos para mejorar su desempeño, lo que representa una fuente de motivación para ellos. Esta evaluación podría implementarse durante todo el proceso educativo del estudiante. Puede ser formal si está legalmente planificada y es esperada en algunos momentos del proceso, o informal si transcurre de manera espontánea, es decir, no planificada. 
Una forma acertada de evaluar este conocimiento es elaborando un cuestionario. La evaluación formativa pudiera ser un examen pequeño o un cuestionario; es un método sencillo para profundizar en el conocimiento de cada estudiante. Se puede evaluar con varios tipos de preguntas: opciones múltiples, completar los espacios en blanco y preguntas con imágenes de respuesta. Lo más práctico es que se puede llevar un registro del progreso y tener acceso directo a las estadísticas (Martínez, 2013).

Al finalizar, es necesario analizar la evaluación sumativa que consiste en evaluar los resultados, e implica una metodología e instrumentos de evaluación para obtener, sintetizar e interpretar la información obtenida; puede ser de evaluación de los aprendizajes, de los efectos o impacto. Recopila descripciones y juicios sobre los resultados alcanzados y establece una relación directa con los objetivos, para comprobar su cumplimiento.

Es aquella compuesta por la suma de valoraciones efectuadas durante un periodo académico, para determinar, al final de este, el grado con que los objetivos de la enseñanza se alcanzaron y así otorgar calificaciones. Estos son acontecimientos de alta significación para la vida de los estudiantes, quienes ocasionalmente pueden percibirlos como barreras a sortear para alcanzar una meta, en lugar de oportunidades para identificar su estado real de aprendizaje.

La evaluación sumativa, también conocida como evaluación de resultado o de impacto, se desarrolla al final de la aplicación de la intervención y se emplea para emitir criterios sobre el programa y sobre su justificación. Se complementa con la evaluación formativa, la cual se lleva a cabo durante el desarrollo del programa para el perfeccionamiento de este, mediante la retroalimentación constante. Por lo antes expuesto, se puede afirmar que es tan importante la evaluación formativa, como la evaluación sumativa, conocida como evaluación de los resultados finales. Sobre esta última se debe mencionar que requiere: estandarización, objetividad y tiempos estrictos (Sánchez, 2015).

Algunas de sus características son:

- Tiene carácter cuantitativo: lleva una calificación.

- Enfatiza los logros y determina el valor del producto final.

- Suele ser realizada en forma individual.

- Pretende comprobar si se han adquirido los conocimientos evaluados.

- Alcanza función acreditativa ya que certifica los conocimientos alcanzados.

\subsection{Gestión de la evaluación del aprendizaje en el EVA}

La evaluación del aprendizaje en el EVA es un proceso que se ha convertido en el centro de preocupación y ocupación de todos los docentes; por lo que es necesario planificar instrumentos evaluativos que partan de las características del curso; el docente se encuentra con que tiene que cambiar algunas apreciaciones sobre sus estudiantes, que obtendría de forma directa o en la enseñanza presencial, con otras herramientas que le facilita el sistema. Como es bien sabido, en 
los EVA el docente dispone de gran cantidad de información que envían sus estudiantes y que le permiten llevar a cabo una evaluación continua del proceso de aprendizaje.

Sobre este tema, en la bibliografía consultada se ha podido constatar que existen diferentes formas para caracterizar la evaluación en el EVA. De manera general, se concibe como el modelo generado y operado en una computadora, que simula o reproduce un entorno real, en sus aspectos más importantes, de acuerdo con la finalidad de su utilización.

En el contexto educativo, la evaluación en el EVA posibilita la comunicación y constatación de resultados, ya sea a través de recursos auditivos y visual, así como su registro temporal o permanente, de manera sincrónica, relacionándose directamente con el tiempo; y asincrónicamente, o sin existir relación con el tiempo, para apoyar dicho proceso a distancia o en línea. Las ventajas de este enfoque son varias, entre la que se identifican las siguientes, de acuerdo con Naidu (2003):

- Motiva el desarrollo de destrezas importantes en los actuales entornos económicos y sociales, como son la comunicación, el trabajo en equipo y el pensamiento crítico.

- Optimiza costos y tiempos, al permitir el empleo de técnicas para evaluar grupos diversificados y con mayor matrícula.

- Posibilita el incremento de nuevas formas de evaluación y su vinculación con otras actividades del aprendizaje, así como una retroalimentación de los resultados.

- Brinda mayores oportunidades para practicar los aprendizajes y habilidades adquiridas.

Desde otra perspectiva, se plantea una idea más concreta, que aparece relacionada con cualquier escenario de evaluación y que está en la base necesaria para decidir adecuadamente sobre qué, cómo, cuándo, dónde y con qué evaluar y cómo informar los resultados. Sin duda, los desafíos que se plantean cuando se aborda esta temática son claros y, en sí, constituyen escenarios para la reflexión y la investigación, además de ser ámbitos que permiten a los docentes la toma de decisiones a partir de los resultados académicos de los estudiantes.

Se hace referencia a un espacio para la reflexión y la investigación, que supone la apertura a nuevas formas de pensar, fundamentar y sentir los espacios de la educación, muchos de ellos presentes en la actualidad. De esta forma se aparecen retos que se deben ser tratados con optimismo y con toda la confianza en su solución, tales como: los problemas para la determinación de los educandos, las oportunidades que nos ofrece el contenido, las interrelaciones que existen entre los agentes implicados, las áreas que pueden y construyen la enseñanza y la evaluación en sí, que implica la determinación de las herramientas a utilizar (Barberá, 2006).

Resulta necesario contar con evaluadores capacitados y que implementen buenas prácticas evaluativas, lo que conlleva a fundamentar, sistematizar y concretar el punto de partida para la toma de decisiones, de los que destacamos: el EVA, donde quedan registrados los aprendizajes del alumnado (comunicación, contenidos, información y recursos), la función que tiene la evaluación (la evaluación como examen y como formación), el contenido que se desea evaluar 
(conceptos, hechos, modos y maneras de tratar, partiendo de los conocimientos específicos y principios generales) y los instrumentos que se necesitan para llevar a cabo dicha evaluación.

\subsection{Instrumentos para la evaluación del aprendizaje en el EVA}

La influencia de la tecnología en los modos de aprender trae consigo nuevos retos para la evaluación. Con el desarrollo de las TIC se han experimentado nuevas maneras de evaluar y diversos son los instrumentos que contribuyen al mejoramiento de este proceso. Es importante reconocer que, en aquellos espacios dirigidos por la tecnología, la evaluación debe verse como un proceso continuo, al igual que la utilización de diferentes instrumentos que posibiliten apreciar el avance de cada alumno en los distintos niveles aprendizaje, por los que transita el estudiante universitario en la construcción de su conocimiento.

EI EVA ofrece diversidad de posibilidades y propicia la adopción de distintos tipos de estrategias docentes, para la aplicación de la evaluación. Aquello instrumentos de evaluación del aprendizaje aplicables en este contexto, alcanzan un valor significativo como elementos facilitadores de la interacción entre docentes y estudiante. Existen variados recursos para la evaluación del aprendizaje en entornos virtuales.

Cada docente podrá fundamentar y seleccionar los instrumentos que puede emplear en los EVA durante el proceso de evaluación del aprendizaje. La selección de un tipo particular guarda relación con la toma de decisiones que toma el profesor durante la planificación y desarrollo de la evaluación. Dadas estas consideraciones, se presentan algunas propuestas como instrumentación de las situaciones de evaluación, las que, a su vez, pueden realizarse en diferentes escenarios.

1. Prueba objetiva: este instrumento es muy común en los entornos virtuales, ya que su ventaja está dada por la posibilidad de su calificación en forma automatizada, posibilitando, además, la recolección de un banco de datos de este tipo de pruebas.

2. Preguntas intercaladas: esta técnica de evaluación se puede emplear durante el desarrollo de una clase ya sea tradicional o a lo largo de la ejecución de los módulos de la educación virtual.

3. Prueba adaptativa y autoadaptadas: la primera son pruebas individuales según el nivel de conocimiento y habilidad alcanzada por el estudiante; en la segunda, el alumno selecciona el nivel de dificultad de cada una de las preguntas que se le presentan. Ambos casos son considerados pruebas objetivas.

4. Mapa conceptual: este instrumento de evaluación permite representar, identificar, relacionar y visualizar conceptos. Se puede utilizar en la enseñanza online, aunque no está muy difundido su uso. Entre los indicadores que el docente debe tener en cuenta para su seguimiento y evaluación, se destacan la jerarquización, que se refiere a la ordenación de los conceptos y el nivel de profundización en el contenido.

5. Foros: tienen gran valor pedagógico ya que permite la interacción haciendo uso del Internet, donde se desarrolla el debate, la concertación y el consenso de idea. Permiten trabajar de manera asincrónica con los alumnos, así como, visualizar y acompañarlos durante la construcción del conocimiento. 
6. Cuestionarios: además de ser flexibles, son también una forma práctica de obtener información. De forma rápida y muy fácil se puede obtener respuestas con herramientas online y móviles. Entre las ventajas fundamentales de este instrumento es la posibilidad que ofrece hacer tantas preguntas como el docente desee.

7. E-portafolios: es una evaluación abierta y flexible, donde los profesores y alumnos establecen los objetivos de aprendizaje. Se conoce también como como un cuaderno de trabajo donde se expone el proceso de aprendizaje a través de la recopilación de evidencias de ese trayecto. Como instrumento de evaluación posee diversas aplicaciones. Facilita que el alumno sea protagonista de su aprendizaje y monitoree sus avances y deficiencias.

8. Rúbricas: este instrumento es muy utilizado por los docentes. Su importancia radica en que permite la gestión y sistematización durante todo el proceso evaluativo. Se debe añadir que facilita la descripción de los criterios a seguir para valorar el trabajo realizado por los estudiantes.

9. Presentaciones o exposiciones a través de videoconferencias: como su nombre lo indica se puede ejecutar mediante el sistema de videoconferencia de escritorio o un sistema institucional; pero lo más importante es que la información se puede ofrecer mediante una presentación oral por parte de los alumnos, o mediante una entrevista.

10. Listas de control: son listas de categorías prefijadas. Se direcciona principalmente a la obtención de información sobre actividades, conductas manifiestas e indicadores. Como aspecto contradictorio debe resaltarse que solo registra presencia o ausencia de la característica observada, no se registran comentarios sobre la conducta, ni el grado posible o razones de su ausencia o presencia.

11. Registros anecdóticos: se emplean mediante la relatoría de conductas relevantes ocurridos en tiempo presente o con carácter retrospectivo Describen los procesos con un nivel de detalle superior en relación a otros instrumentos. Se emplean con la finalidad de identificar líneas de conductas más o menos estables, para proporcionar evidencias sobre los cambios o ausencias de cambios en los estudiantes.

12. Diarios de clases: son registros escritos retrospectivamente de la conducta personal o de otros en forma continua o regular. Pueden adoptar diferentes formas: abiertos o cerrados (Lezcano \& Villanova, 2017).

\section{Metodología}

El trabajo presentado se realizó desde el empleo de una metodología cualitativa descriptiva, teniendo en cuenta un diseño flexible que considera el proceso investigativo en forma circular y en orden creciente de menor a mayor complejidad. Desde el momento en que se concibe y considera este proceso, fue posible reconocer nuevas e inesperadas situaciones, relacionadas con el objeto de investigación, durante todo el recorrido investigativo. Dadas las posibilidades que ofrece la perspectiva cualitativa, se emplearon algunos métodos científicos y técnicas de recolección de datos. Teniendo en cuenta la importancia del tema, se plantearon las siguientes interrogantes de la investigación, las cuales permitieron su curso y la determinación de los objetivos: ¿Qué metodología utilizar durante la investigación? ¿Cuáles son los fundamentos teóricos sobre la

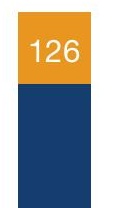


evaluación del aprendizaje? ¿Cómo gestionar la evaluación del aprendizaje desde el EVA en el contexto universitario actual? ¿Qué criterios manifiestan los docentes sobre la evaluación del aprendizaje desde el EVA en el contexto universitario?

El objetivo general de la investigación se centra en reconocer la importancia de la evaluación del aprendizaje desde el EVA en el contexto universitario actual. A su vez, fueron determinados como objetivos específicos el determinar la metodología a utilizar durante el proceso investigativo, fundamentar teóricamente la evaluación del aprendizaje, argumentar la gestión de la evaluación del aprendizaje desde el EVA en el contexto universitario actual e indagar en el criterio de algunos docentes sobre la evaluación del aprendizaje desde el EVA en el contexto universitario.

Dando cumplimento a los objetivos específicos, y llegar al cumplimiento del objetivo general, las acciones investigativas se planificaron desde tres momentos que se desarrollaron durante todo el proceso investigativo, y que serán descritas. Para su cumplimiento, se emplearon diferentes métodos de investigación que posibilitaron a los autores desarrollar un conjunto de procedimientos lógicos. Además, su empleo oportuno y adecuado favoreció la argumentación y la demostración en función del objeto de investigación.

En relación al método del nivel teórico se utilizó el analítico - sintético, este favoreció el desarrollo del análisis y la síntesis, a partir del estudio de la teoría sobre la temática abordada. Al mismo tiempo, a través de la síntesis se evidencia su integración en su interrelación como un todo. Este método contribuyó al desarrollo de la fundamentación teórica de la investigación, permitiendo llegar a la descomposición de los diferentes criterios sobre la evaluación del aprendizaje y su gestión desde el EVA en el contexto universitario.

A su vez, en cuanto a los métodos del nivel empírico se empleó la encuesta, esta posibilitó la recopilación de datos y el criterio de los docentes sobre la importancia de la evaluación y su gestión en el EVA en el contexto universitario. Los datos se obtuvieron al realizar un grupo de preguntas normalizadas encaminadas directamente a la muestra representativa, seleccionada anteriormente.

El análisis porcentual fue aplicado como método del nivel estadístico. Mediante este se desarrollaron los procedimientos pertinentes para el manejo de datos cuantitativos, reflejando el resultado de la encuesta aplicada como técnica de recolección y su análisis, permitiendo así la representación gráfica de los resultados.

Finalmente, se aplicó el método del nivel lógico de la investigación científica de manera inductiva, el cual permitió a los autores el razonamiento, partiendo de casos particulares en la utilización de la evaluación del aprendizaje en el EVA, desde el contexto universitario, para llevarlo a conocimientos generales sobre esta temática, lo que permitió llegar a las conclusiones de la investigación, logrando así el cumplimiento del objetivo general.

\section{Resultados}


El proceso investigativo tuvo lugar desde la concepción de tres momentos fundamentales: diseño del marco metodológico, elaboración de los fundamentos teóricos y la aplicación de una encuesta a docentes universitarios seleccionados. Cada momento estuvo guiado por la determinación de los objetivos que se corresponden con los objetivos específicos de la investigación y los procedimientos desarrollados, permitiendo así el cumplimiento del objetivo general planificado.

Se considera pertinente explicar que los momentos del proceso de la investigación forman parte de un mismo proceso y se dan en forma conjunta. La función de base es la exploración, momento en que los investigadores pudieron adoptar nuevas perspectivas, avanzar y volver sobre aspectos o momentos que ya estaban delimitados, a medida que se avanzaba en complejidad e interpretación del objeto investigado. La apertura a las situaciones que se fueron gestando en el proceso y la actitud creativa para asumir posibles caminos de búsqueda, fueron condicionante fundamental para iniciar el recorrido de esta investigación cualitativa.

- Primer momento: determinación del marco metodológico de la investigación.

\section{Objetivo:}

- Determinar la metodología a utilizar durante el proceso investigativo.

\section{Procedimientos:}

- Planificación de los tres momentos del proceso investigativo.

- Planificación del objetivo general y los objetivos específicos.

- Determinación la metodología a emplear.

\section{Descripción:}

Este momento se corresponde con la planificación de la investigación. Inicialmente los autores del presente trabajo planificaron los momentos en los que se ha estructurado el proceso investigativo, centrando la atención en la evaluación del aprendizaje en el EVA, desde el contexto universitario actual. Seguidamente se determinó el objetivo general y los objetivos específicos, los cuales se corresponden con los objetivos que se toman en cuenta para dar cumplimiento al proceso investigativo en cada uno de estos momentos. Para la presentación de los resultados, en un primer momento se sigue una metodología cualitativa descriptiva, y después se aplica el cálculo porcentual, método del nivel estadísticos que se utilizó para describir e ilustrar los resultados de la encuesta aplicada a los docentes. De manera general, en este primer momento de la investigación se seleccionan los métodos utilizados, los cuales fueron cumplidos y descritos anteriormente.

- Segundo momento: determinación del marco teórico de la investigación.

\section{Objetivos:}

- Fundamentar teóricamente la evaluación del aprendizaje.

- Argumentar la gestión de la evaluación del aprendizaje desde el EVA en el contexto universitario. 


\section{Procedimientos:}

- Estudio de la bibliografía que guarde relación con el tema de investigado.

- Determinación de inferencias en cuanto a los diferentes criterios sobre la evaluación del aprendizaje, con énfasis en el EVA desde el contexto universitario.

- Elaboración del marco teórico del artículo.

\section{Descripción:}

Dando continuidad al proceso investigativo, y así cumplir con los objetivos planificados para el segundo momento, los autores de la investigación consideran pertinente explicar que la realización del presente artículo, inicialmente se desarrolló por medio de la investigación documental, asumiéndose como un proceso sustentado en la búsqueda, recuperación, análisis, crítica e interpretación de la información obtenida en diferentes fuentes impresas o electrónicas, en la búsqueda de nuevas ideas.

Tomando como referente este criterio, se consultaron diferentes autores que han dado su aporte en el plano de la teoría sobre el objeto de estudio, con la finalidad de demostrar la validez del contenido. De esta manera, se logró el cumplimiento del objetivo general del estudio, destacando la importancia que tiene la evaluación del aprendizaje desde EVA en un contexto universitario. El análisis practicado permitió recabar las evidencias documentales que permitieron corroborar y avalar los contenidos.

El análisis de los fundamentos teóricos relacionados con la investigación evidencia que la evaluación en el EVA debe estar apoyada en procesos autoinstructivos, para ello se requiere una atención especial a la autoevaluación, teniendo en cuenta que durante la presencialidad es el docente quien tradicionalmente se encarga de evaluar a los estudiantes durante las infinitas interacciones. Teniendo en cuenta la variedad de características de los EVA, se debe aludir a las grandes diferencias entre desarrollar procesos evaluativos en este contexto y ejecutarlo en los ambientes resultantes de la presencialidad.

Con relación a la evaluación según su funcionalidad u objetivo en el EVA, se destaca que los profesores obtienen información de sus alumnos de un modo sencillo y rápido, descargando la actividad automática de corrección y administración de comentarios en manos del ordenador, y los alumnos obtienen una herramienta de aprendizaje, ya que tienen la oportunidad de medir sus conocimientos durante el periodo académico y conocer sus fallas y debilidades.

A partir del criterio de los autores consultados se pudo inferir que el cambio significativo se ha generado sólo en el medio, a través del cual se evalúa al estudiante, no evidenciándose este comportamiento desde la concepción de la evaluación, pues en ésta puede reflejarse una visión mecanicista, medible y reproductora, dependiendo cómo se concibe la evaluación de los aprendizajes en los estudios en línea. 
Esta situación señala la necesidad de descubrir, proponer y practicar nuevas formas para convertir a la evaluación radicalmente diferente en el EVA, sin que esto implique el traslado de los modelos de evaluación presencial hacia los contextos virtuales.

- Tercer momento: aplicación de una encuesta a docentes universitarios.

\section{Objetivo:}

- Indagar en el criterio de algunos docentes sobre la evaluación del aprendizaje desde el EVA en el contexto universitario actual.

\section{Procedimientos:}

- Elaboración de la encuesta.

- Aplicación de la encuesta después de seleccionada la muestra.

- Análisis de los resultados obtenidos.

- Redacción de las conclusiones.

\section{Descripción:}

Ya en el tercer y último momento de la investigación se procedió a la elaboración de una encuesta (ver Anexo 1), cuyo objetivo se enmarca en comprobar el estado de opinión de los docentes universitarios sobre la importancia de la evaluación del aprendizaje en el EVA. Para su aplicación, se tuvo en cuenta una población de 54 profesores universitarios que impartieron docencia en el período académico abril 2020 - septiembre 2020, de los cuales se seleccionó una muestra de 20 docentes, representando el $37,07 \%$.

El análisis de los resultados permitió corroborar que el 100\% de los docentes encuestados consideran muy importante el proceso de evaluación del aprendizaje de sus estudiantes en el EVA. El 65\% manifestó poseer una vasta experiencia en la evaluación del aprendizaje de sus estudiantes desde EVA, antes del confinamiento, por la situación sanitaria que vive el Ecuador; el $20 \%$ expresó poseer una experiencia suficiente para su desempeño en este sentido, mientras que el $15 \%$ declaró no tener experiencia para estos nuevos retos, de lo que infiere, que, en algunos casos, el tema debe ser centro de atención tanto en el ámbito personal, como para algunas instituciones.

Otra de las preguntas de la encuesta arrojó que el $75 \%$ conoce varios instrumentos para evaluar el aprendizaje de sus estudiantes en el EVA; solo el $25 \%$ manifestó conocer algunos de estos instrumentos. Relacionado con su utilización, el $60 \%$ declaró variarlos siempre y el $40 \%$ indicó variar su utilización casi siempre. Estos resultados posibilitaron el análisis de que existe un 15\% de los docentes encuestados que conocen varios instrumentos de evaluación del aprendizaje en el EVA, pero en el momento de emplearlos en sus clases, no siempre los varían. 
Relacionado con los momentos en que los profesores implementan la evaluación del aprendizaje, se pudo determinar que el $75 \%$ coincide en implementarla durante el desarrollo de la clase y como actividad independiente fuera de la misma; mientras que solo $15 \%$ expresa implementarla durante el desarrollo de la clase, y el $10 \%$ la utiliza como actividad independiente fuera de la esta.

A partir de los resultados expuestos, los autores de la investigación infieren que los docentes encuestados reconocen la importancia del proceso de evaluación del aprendizaje de sus estudiantes en el EVA. En su mayoría, poseen una vasta experiencia para su implementación, lo que se evidencia al manifestar que conocen y utilizan varios instrumentos evaluativos, así como los momentos en que debe aplicarse la evaluación del aprendizaje. La presentación de los resultados descritos anteriormente permitió comprobar el cumplimiento del objetivo general, arribando a las conclusiones finales.

\section{Conclusiones}

La investigación que se presenta tuvo lugar desde la planificación de tres momentos fundamentales: diseño del marco metodológico, elaboración de los fundamentos teóricos y la aplicación de una encuesta a docentes universitarios.

La utilización de diferentes métodos permitió el desarrollo del proceso investigativo en cada uno de estos momentos; empleándose inicialmente una metodología cualitativa descriptiva para la exposición de los resultados y para finalizar fue practicado el análisis porcentual.

La consulta bibliográfica permitió corroborar el criterio de varios autores, quienes coinciden al manifestar que la evaluación es la emisión de un juicio de valor sobre algo que se quiere mejorar, teniendo en cuenta tres momentos fundamentales: recogida de información, análisis de dicha información y toma de decisiones de acuerdo con el juicio emitido.

Se presentan diferentes instrumentos para la evaluación del aprendizaje en el EVA, tomando como referente que esta posibilita la comunicación y constatación de los resultados de aprendizaje.

Teniendo en cuenta las características tan variadas del EVA, se reconoce una gran diferencia al desarrollar procesos evaluativos en este contexto y al hacerlo en los ambientes del aula con carácter presencial; tal diferencia se enmarca desde el momento en que se concibe la evaluación de los aprendizajes en los espacios virtuales.

La revisión documental practicada durante el proceso investigativo y el análisis de los fundamentos teóricos demostraron la importancia de la evaluación desde el EVA, en el contexto universitario actual, y a su vez, la encuesta aplicada a los docentes universitarios corroboró el cumplimiento del objetivo general de la investigación, al destacar la utilización de la evaluación en el EVA como nuevo escenario pedagógico, valorando así su importancia. 


\section{Referencias}

Barberá, E. (2016). Aportaciones de la tecnología a la e-Evaluación. Revista de Educación a distancia (RED), (50). http://dx.doi.org/10.6018/red/50/4

Bélair, L. M. (2000). La evaluación en la acción: el dossier progresivo de los alumnos. Díada.

Casanova, M. A. (2012). El diseño curricular como factor de calidad educativa. Revista Iberoamericana sobre Calidad, Eficacia y Cambio en Educación, 10(4) 4-18. https://revistas.uam.es/reice/issue/view/345/194

Castillo, S., \& Cabrerizo, J. (2003). Evaluación educativa y promoción escolar. Pearson Educación.

Jorba, J., \& Sanmartí, N. (1996). Enseñar, aprender y evaluar

un proceso de regulación continua: propuestas didácticas para las áreas de ciencias de la naturaleza y matemáticas. Ministerio de Educación Cultura y Deporte. https://dialnet.unirioja.es/servlet/libro?codigo=186191

Lezcano, L., \& Vilanova, G. (2017). Instrumentos de evaluación de aprendizaje en entornos virtuales. Perspectiva de estudiantes y aportes de docentes. Informes Científicos Técnicos-UNPA, 9(1), 1-36. https://dialnet. unirioja.es/servlet/articulo?codigo $=5919087$

Martínez Rizo, F. (2009). Evaluación formativa en aula y evaluación a gran escala: hacia un sistema más equilibrado. Revista electrónica de investigación educativa, 11(2), 1-18. http://redie.uabc.mx/redie/article/ view/231

Martínez Rizo, F. (2013). Dificultades para implementar la evaluación formativa: Revisión de literatura. Perfiles educativos, 35(139), 128-150. http://www.scielo.org.mx/scielo.php?pid=S0185-26982013000100009\&script=sci abstract\&tlng=pt

Naidu, S. (2003). Learning and Teaching with Technology. London and Sterling, VA. https://doi. org/10.4324/9780203416891

Ploegh, K., Tillema, H., \& Segers, M. (2009). In search of quality criteria in peer assessment practises. Studies in Educational Evaluation, 35(2-3) 102-109. https://doi.org/10.1016/j.stueduc.2009.05.001

Sánchez, M. (2015). Educación médica. Teoría y práctica. Elsevier. https://www.elsevier.com/books/educacion-medica-teoria-y-practica/sanchez-mendiola/978-84-9022-778-7

Zaragoza Raduà, J. M. (2005). Actitudes del profesorado de secundaria obligatoria hacia la evaluación de los aprendizajes de los alumnos. [Tesis doctoral, Universitat Autònoma de Barcelona] https://hdl.handle. net/10803/5023 


\section{Anexos}

\section{Anexo 1}

\section{Encuesta aplicada a los docentes universitarios seleccionados}

Objetivo: Comprobar el estado de opinión de los docentes universitarios encuestados sobre la importancia de la evaluación del aprendizaje en el EVA.

Lea detenidamente cada una de las preguntas y marque la opción que considere pertinente. Agradecemos su participación y su contribución al desarrollo de esta investigación.

1- ¿Cómo considera el proceso de evaluación del aprendizaje de sus estudiantes en el EVA?
a) ___ Muy importante
b) ___ Importante
c) ___ Poco importante

2- Califique su experiencia docente en la evaluación del aprendizaje de sus estudiantes desde EVA, antes del confinamiento por la situación sanitaria que vive el Ecuador:
a) ___ Basta experiencia
b) ___ Suficiente experiencia
c) ___ Sin experiencia

3- ¿Conoce los instrumentos para evaluar el aprendizaje de sus estudiantes en el EVA?
a) Varios
b) ___ Algunos
c) ___ Ninguno

4- ¿Con qué frecuencia varía usted los instrumentos para evaluar el aprendizaje de sus estudiantes en el EVA?
a) Siempre
b) ___ Casi siempre
c) ___ Nunca

5- La evaluación del aprendizaje de sus estudiantes en el EVA se manifiesta:
a) ___ Durante el desarrollo de la clase y como actividad independiente fuera de la clase.
b) ___ Durante el desarrollo de la clase.
c) ___ Como actividad independiente fuera de la clase. 
Copyright (c) 2021 Arian Fuentes Aparicio, Betty Pastora Alejo, Ariadna Granados Campo y Osley Puerto Menéndez

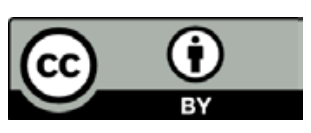

Este texto está protegido bajo una licencia internacional Creative Commons 4.0.

Usted es libre para Compartir-copiar y redistribuir el material en cualquier medio o formato - y Adaptar el documento - remezclar, transformar y crear a partir del material-para cualquier propósito, incluso para fines comerciales, siempre que cumpla las condiciones de Atribución. Usted debe dar crédito a la obra original de manera adecuada, proporcionar un enlace a la licencia, e indicar si se han realizado cambios. Puede hacerlo en cualquier forma razonable, pero no de forma tal que sugiera que tiene el apoyo del licenciante o lo recibe por el uso que hace de la obra.

\section{$\underline{\text { Resumen de licencia - Texto completo de la licencia }}$}

